\title{
EFFECT OF TWO DIFFERENT IMPRESSION MATERIALS ON THE ACCURACY OF THE MASTER CASTS OF PARTIALLY EDENTULOUS CASES (IN-VITRO STUDY)
}

\author{
Shaimaa Lotfy* and Yasmine G. Thabet**
}

\begin{abstract}
Objectives: This study was conducted to assess and compare the effect of storage time on the dimensional stability of extended-pour alginates and polyether impression materials.

Materials and Methods: This study was applied on Kennedy class II mod 1 mandibular educational model. Impressions of the model were made using Hydrogum 5 (extended pour alginate) and Impregum polyether material. Four groups were defined according to the impression material and the pouring time: Group I: Impressions were made using extended pour alginate and were poured after one day. Group II: Impressions were made using polyether impression material and were poured after one day. Group III: Impressions were made using extended pour alginate and were poured after five days. Group IV: Impressions were made using polyether impression material and were poured after five days. For each group, ten different impressions were prepared. The impressions were poured to create the casts that were digitally scanned. A 3D data analyzing software was used to evaluate the presence of discrepancies between the original cast and the experimental casts.
\end{abstract}

Results: Group III showed the highest value $0.91 \pm 0.07$, regarding average deviation from the defined horizontal plane in the reference cast, followed by group I $0.05 \pm 0.037$, and group IV $0.25 \pm 0.019$ while group II showed the lowest value $0.02 \pm 0.012$. Kruskal Wallis test showed significant difference between the studied groups and Mann Whitney test with Bonferrioni correction showed statistically significant difference between Group III with group II and group IV.

Conclusion: Under appropriate storage conditions, polyether impression material poured after one day produced the most dimensionally accurate casts however, extended pour alginates attained clinically acceptable range of dimensional accuracy.

KEY WORDS: accuracy of impressions, extended-pour alginates, polyether.

* Associate Professor of Removable Prosthodontics, Faculty of Dentistry Ain Shams University.

** Lecturer of Removable Prosthodontics, Faculty of Dentistry Ain Shams University. 


\section{INTRODUCTION}

Accurate impression procedures and precise creation of stone models are critical steps in the construction of successful restorations for oral rehabilitation. Impressions should replicate hard and soft tissues to fabricate biologically, mechanically, functionally and esthetically acceptable prosthesis. The impression material and the technique followed are important elements of impression accuracy. ${ }^{(1)}$

Five main factors may cause dimensional changes of impressions which are polymerization shrinkage, loss of by-products during condensation, thermal contraction from oral temperatures to room temperature, imbibition when exposed to water, disinfectants or high humidity and incomplete recovery of deformation because of viscoelastic behavior. Moreover, storage conditions, delayed or repeated pouring, and distortion of the impressions on retrieval from the casts influence the accuracy of the gypsum models. ${ }^{(2,3,4)}$

Polyethers undergo an addition-cured polymerization reaction on setting which has no by-products resulting in a material with very good dimensional stability. They are highly hydrophilic, thus they are liable to water absorption from the atmosphere or the storage medium. They may also absorb water from the dental stone, which can cause expansion of the moulds and therefore smaller models. Thus, the set impressions may swell and distort due to imbibition of water on storage in high humidity and it is advocated to store the impressions in a dry environment. ${ }^{(3,5,6)}$

Alginate is one of the most frequently used impression materials. Although some professionals use it for definitive impressions due to its good surface details and fast setting, yet problems with its dimensional stability limit its use. Alginates are hydrophilic in nature which facilitates making accurate impressions in the presence of saliva or blood. However, accurate alginate impressions are only achieved when the gypsum models are immediately poured. ${ }^{(4,7)}$
Alginate impressions undergo imbibition and syneresis when left in the normal clinical environment thus the time before pouring them is critical. The impressions should be covered with damp gauze and left in zip-lock plastic bags until the casts are poured. The elastic recovery of alginate impression material is slow, thus pouring them should be delayed for 10 minutes only. However, wrapping the impressions in a wet paper towel can't be an acceptable alternative to immediately pouring the gypsum models. It was reported that the alginate absorbs the moisture from the paper towels as well as uneven weight or pressure from the towels may cause distortion of the impressions. ${ }^{(5,8)}$

A new generation of irreversible hydrocolloids was introduced in the market which is capable of maintaining its dimensional stability for up to 5 days. This invention would change the established workflow of alginate impressions that has been set for decades which dictated that impressions must be immediately poured and never be immersed in liquids, wrapped in damp towels for long periods or stored before pouring them to obtain the gypsum models.

Very few studies investigated the dimensional accuracy of extended-pour alginates ${ }^{(7,9,10)}$ Hence this study was conducted to assess and compare the effect of storage time on the dimensional stability of extended-pour alginates and polyether impression materials poured after one and five days of impression taking.

\section{MATERIALS AND METHODS}

This study was applied on Kennedy class II mod 1 mandibular educational model where the abutments were the first premolar and the second molar on one side and the second premolar on the other side. Impressions of the model were made using Hydrogum 5 (extended pour alginate, Zhermack, Italy) and Impregum polyether material. (3M Espe, Seefeld, Starnberg, Germany). 
Four groups were defined according to the impression material and the pouring time:

Group I: Impressions were made using extended pour alginate and were poured after one day.

Group II: Impressions were made using polyether impression material and were poured after one day.

Group III: Impressions were made using extended pour alginate and were poured after five days.

Group IV: Impressions were made using polyether impression material and were poured after five days.

Ten impressions were made for each group by a single operator.

\section{Preparation of the model}

Three stops were made on the model with autopolymerizing acrylic resin. These stops ensured the achievement of reproducible positioning of the trays during impression making. (Fig.1)

Two layers of spacer wax were placed on the teeth surfaces of the model in order to allow 4-5 $\mathrm{mm}$ space for adequate uniform thickness of the alginate.

One layer of spacer wax was utilized in the trays used for the polyether material.
Custom trays were prepared with autopolymerizing acrylic resin. Perforations in the trays allowed the excessive alginate to extravagate without resistance, allowing complete seating of the tray on the master model.

The manufacturers' instructions were strictly followed during manipulation of the materials. The proper amount of tap water was placed in a mixing bowl and the impression material was added to it. The powder was folded into the water and mixed by hand till the mix was homogenous. The impression material was loaded in the trays and a small amount of material was wiped over the occlusal, facial and lingual surfaces of the abutment teeth. The trays were seated on the model until they rested firmly against the stops.

Impregum polyether was mixed using a glass slab and cement spatula. Equal lengths of base and catalyst paste were dispensed and an impression syringe was used to inject the material around the teeth. After the setting time, the tray was separated from the master model. (Fig. 1)

Following the manufacturer's instructions, the extended-pour alginate impressions were not wrapped in paper towels but simply sealed in plastic zipper storage bags (100\% relative humidity) at room temperature where the air inside the bags was removed as much as possible for the specified

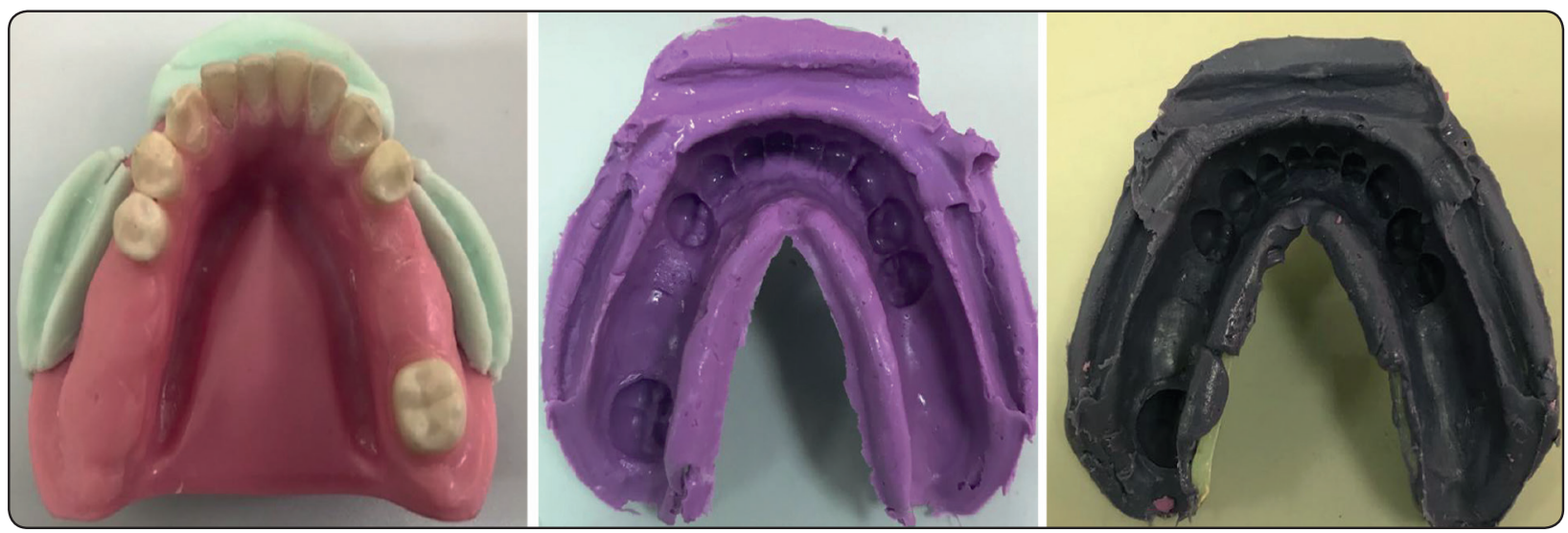

Fig. (1): Original cast, alginate and polyether impressions. 
storage time periods. The same was applied to the polyether impressions.

The impressions were poured at the specified pouring timings to create the models using type III dental stone, according to the manufacturer's recommendations. 140 grams of powder were vacuum mixed with $40 \mathrm{ml}$ of tap water. The stone was then gently vibrated into the impressions. After one hour, the stone casts were separated from the impressions. All casts were stored at room temperature for minimum 24 hours before they were scanned.

The casts were sprayed with scanable spray (Shera scanspray, Werkstoff Technologie, Germany) to reduce the reflection of their surfaces before they were scanned.

The desktop scanner was used to scan the casts and the original model using the 3 shape software. The casts were fixed on a plate inside the scanner with their labial surface facing the inside of the scanner. A STL file of the scanned image of the cast was created and imported to the measurement system.

A 3D data analyzing software (Geomagic Control X) was used to evaluate the presence of discrepancies between STL files of the original cast and the experimental casts. The STL file of the original cast was used as a reference. A horizontal plane was defined on the original cast that was aligned $29 \mathrm{~mm}$ from the base of the cast cross-sectioning the teeth to be used as a reference plane for the measurements of the experimental casts. (Fig. 2)

Ten STL files were obtained from every experimental group. They were matched with the reference file respectively by the best fit algorithm. Selecting and cutting tools inside the software were used on the matched imaging data to eliminate the irrelevant areas. Then the 3D data of the casts were generated for discrepancy analysis through the previously defined horizontal plane on the original cast. (Fig. 3)

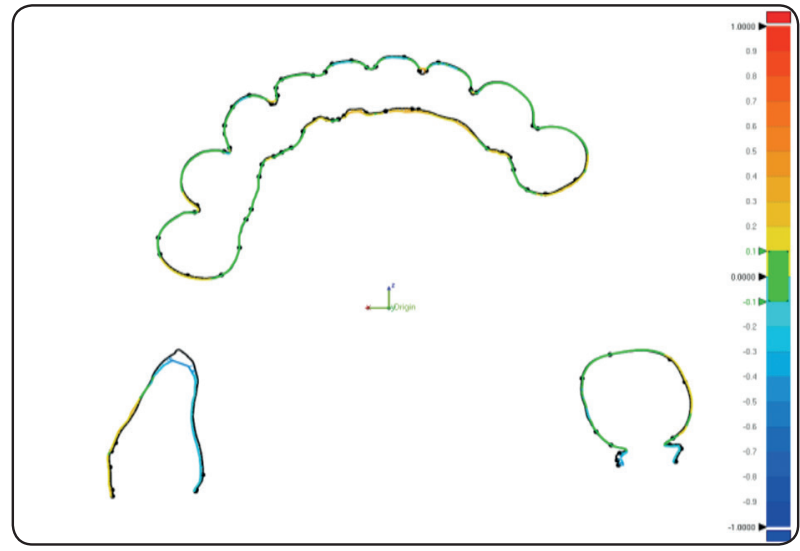

Fig. (2): Horizontal plane defined on the original cast used for measurements of discrepancies in experimental casts.

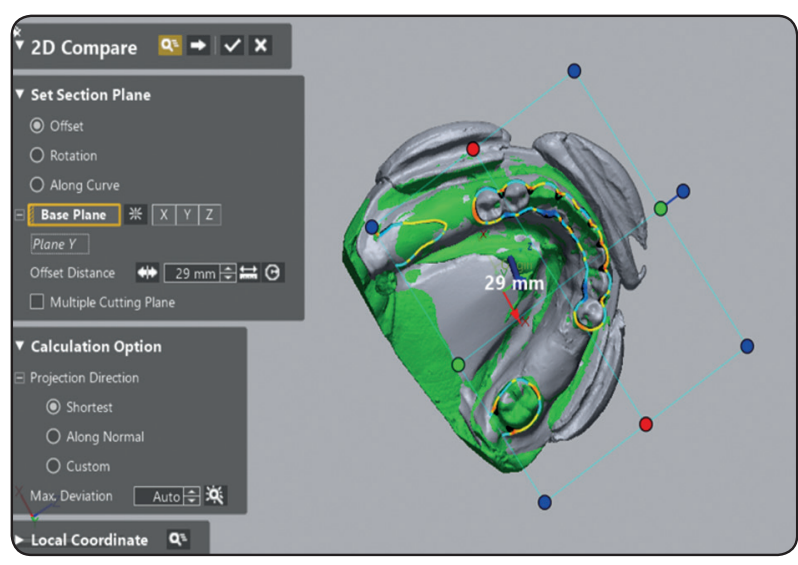

Fig. (3): The experimental and original casts superimposed together with the defined reference horizontal plane.

\section{RESULTS}

Collected data were tested for normality by checking the data distribution, calculating the mean and median values, evaluating histograms and normality curves and using Kruskal Wallis test followed by Mann Whitney test. Data were presented by mean and standard deviation (SD). Kruskal Wallis test showed significant difference between the studied groups. It was followed by pair wise comparison with Mann Whitney test with Bonferrioni correction. The significance level was set at $\mathrm{P} \leq 0.05$. Statistical analysis was performed with IBM ${ }^{\circledR}$ SPSS $\AA$ Statistics Version 20 for Windows. 
TABLE (1): Descriptive statics and $p$ value of average deviation from the horizontal plane in the reference cast.

\begin{tabular}{|c|c|c|c|c|c|c|c|}
\hline & $\mathrm{N}$ & Mean deviation & Std. Deviation & Std. Error & Minimum & Maximum & P value \\
\hline Group I & 10 & $.0520^{\mathrm{ab}}$ & .03706 & .01075 & .010 & .11 \\
\hline Group II & 10 & $.0200^{\mathrm{b}}$ & .01247 & .01958 & .009 & .04 \\
\hline Group III & 10 & $.0910^{\mathrm{a}}$ & .07340 & .00348 & .011 & .24 \\
\hline Group IV & 10 & $.0250^{\mathrm{b}}$ & .01958 & .00755 & .012 & .07 \\
\hline
\end{tabular}

As shown in table (1), regarding average deviation from the defined horizontal plane in the reference cast, group III showed the highest value $0.91^{\circ} \pm 0.07$ followed by group I $0.05 \pm 0.037$, and group IV $0.25 \pm 0.019$ while group II showed the lowest value $0.02^{\circ} \pm 0.012$. Kruskal Wallis test showed significant difference between the studied groups and Mann Whitney test with Bonferrioni correction showed statistically significant difference between group III with group II and group IV.

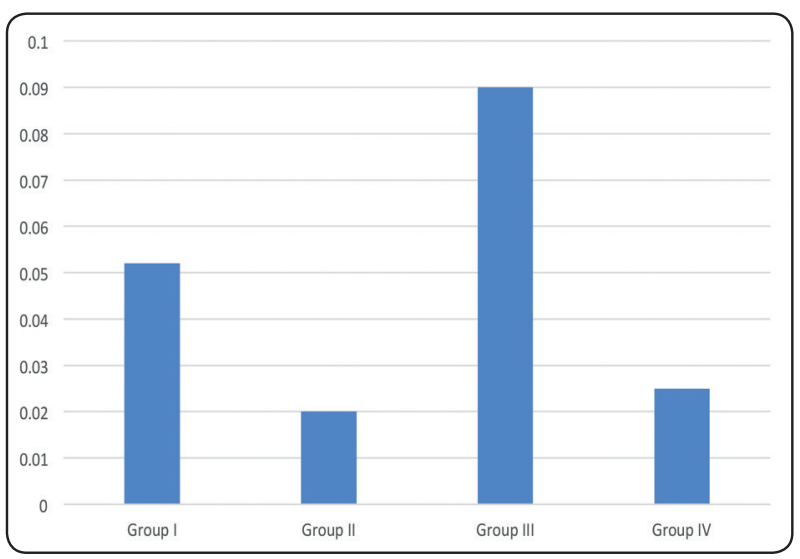

Fig. (4): Mean deviation of the studied groups from the horizontal plane in the reference cast.

\section{DISCUSSION}

Impressions should be accurate and maintain their dimensional stability until poured to create gypsum casts. Accuracy is the ability to reproduce a true measured value while dimensional stability is the ability to maintain accuracy over time. A material may be highly dimensionally accurate after its initial polymerization but becomes less accurate after storage for a period of time. It is critical that an impression material maintains its dimensional accuracy even when pouring of impressions is delayed. (11)

The accuracy of an impression is determined by two factors which are trueness and precision. Trueness indicates the deviation of the tested impression from the original geometry. On the other hand, precision describes the deviations between the impressions in a test group. Both describe all aspects of accuracy of a specific impression technique. ${ }^{(12,13)}$ In this study trueness was evaluated by detection of average deviations in the experimental casts from the previously defined horizontal plane in the original model where both models were superimposed together using the software.

The dimensional changes of the two impression materials after one day and after five days are multifactorial and material specific. Factors influencing the impression materials include syneresis, effect of free water loss or gain via evaporation and imbibition, ratios of calcium to sodium and fillers to polymers, molecular weight of alginic polymers and other proprietary constituents. However, dimensional changes related to syneresis are usually controlled to a great extent by the 
manufacturer of the impression materials as they are influenced by the constituents of the alginate. ${ }^{(2-6)}$ Thus, extended storage time may cause dimensional changes particularly in alginate impressions. ${ }^{(14,15)}$

Random errors may occur while making impressions and generating gypsum casts. Such errors include incorrect ratios of gypsum powder to water, impression material may be unsupported by the tray, movement of the tray during setting, debonding from the tray, incorrect removal of the tray from the mouth and prolonged contact of the impression material with the gypsum product. Gypsum products exhibit a net expansion during setting. ${ }^{(8)}$

Controversies exist regarding the clinically acceptable range of dimensional accuracy which varies $0.1 \%$ to $0.8 \%{ }^{(16)} \mathrm{A}$ study reported the amount of distortion during opening the mandible and impression making ranges from 0.1 to $0.5 \mathrm{~mm}$. Similar to previously performed studies, the mean values $(0.5 \%)$ were considered as the standard threshold for maximum permissible dimensional changes. ${ }^{(9,10,16)}$

In this study, polyether impression material gave the most accurate results. This coincides with several previous studies that revealed that polyethers were more accurate than the other materials. ${ }^{(11,17,18)}$ This may be attributed to their addition-cured polymerization reaction on setting which has no by-products resulting in very good dimensional stability. Although, the extended pour alginates showed significantly higher deviations than polyether when poured at one and five days, yet it had clinically acceptable dimensional changes at all pouring times. These results coincide with previous studies on extended pour alginates. ${ }^{(7,9,10,19,20)}$

In this study, there are some limitations, as this experiment could not be considered identical to pouring a cast from intra-oral impressions due to absence of the effect of oral fluids, soft tissues and different arch forms. Thus, there is scope for further researches taking these factors into consideration.

\section{CONCLUSION}

Under appropriate storage conditions, polyether impression material poured after one day produced the most dimensionally accurate casts however, extended pour alginates attained clinically acceptable range of dimensional accuracy.

\section{REFERENCES}

1- Walker M.P., Ries D., Borello B.: Implant cast accuracy as a function of impression techniques and impression materials viscosity, Int. J. Oral Maxillofac. Implants, 2008 23(4):669-74.

2- Cynthia S.P., Mary P.W., Aisling M., and Paulette S.: Dimensional accuracy and surface detail reproduction of two hydrophilic vinyl polysiloxane impression materials tested under dry, moist, and wet conditions, J Prosthet. Dent, 2003, 90:365-72

3- Anusavice J.K.: Phillips' science of dental materials, 12th edition. Elsevier. Pages; 2012.

4- Chen S.T., Liang W.M., Chen F.N.: Factors affecting the accuracy of elastomeric impression materials. J of Dentistry, 2004, 32, 603-609.

5- Vasconcellos F.E., Andreullo R.F., Sabrosa C.E., Neisser M.P., Rego MA.: Dimensional stability of casts obtained with polyether and addition reaction silicone after disinfection with sodium hypochlorite and peracetic acid., Rev Bras Odontol ,2012;1: 55-60.

6- Rodriguez J.M., Bartlett D.W.: The dimensional stability of impression materials and its effect on in vitro tooth wear study, Dent Mater, 2011,27:253-58.

7- $\quad$ Todd J.A., Oesterle L.J., Newman S.M., Shellhart W.C.: Dimensional changes of extended-pour alginate impression materials, Am. J. Orthod. Dentofacial Orthop., 2013 , 143(4): 55-63.

8- Ashley M.: Making a good impression: A “How to' Paper on dental 1. Alginate, Dent Update 2005,32:169-75.

9- Rohanian A., Shabestari1 G.O., Zeighami S., Samadi M.J., Shamshiri A.R.: Effect of Storage Time of Extended-Pour and Conventional Alginate Impressions on Dimensional Accuracy of Casts, J Dent (Tehran), 2014, 11(6): 655-664.

10- Imbery T.A., Nehring J., Janus C., Moon P.C.: Accuracy and dimensional stability of extended-pour and conventional alginate impression materials, JADA, 2010, 141(1):32-39. 
11- Neethu L., Vasunni G. K.: Comparative Evaluation of Dimensional Stability of Three Different Elastomeric Impression Materials - An Invitro Study, J Dent and Medical Sciences, 2015,14(9): 89-93

12- Chandran D.T., Jagger D.C., Jagger R.G., Barbour M.E.: Two- and three-dimensional accuracy of dental impression materials: effects of storage time and moisture contamination, Biomed Mater Eng, 2010, 20(5):243-249.

13- Ziegler M.: Digital impression taking with reproducibly high precision, International journal of computerized dentistry, 2009, 12(2):159-163.

14- Ozkalayci N., Koroglu A.,Borekci C.: Storage condition and period effect on the dimensional stability of irreversible hydrocolloid impression materials, Cumhuriyet Dent. Journal, 2017,20(1):30-39.

15- Rodrigues S.B., Augusto C.R., Leitune V.C., Samuel S.M., Collares F.M.: Influence of delayed pouring on irreversible hydrocolloid properties, Braz. Oral Res., 2012, 26(5): 404-9.
16- Erbe C., Ruf S., Wöstmann B., Balkenhol M.: Dimensional stability of contemporary irreversible hydrocolloids: humidor versus wet tissue storage, J. Prosthet Dent., 2012, 108(2):114-22.

17- Faria A., Rodrigues R., Macedo A., Chiarello de Mattos M., Ribeiro R.: Accuracy of stone casts obtained by different impression materials, Braz Oral Res., 2008, 22(4):293-8.

18- Bajoghli F., Sabouhi M., Nosouhian S., Davoudi A., Behnamnia Z.: Comparing the Accuracy of Three Different Impression Materials in Making Duplicate Dies, J. International Oral Health, 2015, 7(7):12-16.

19- Walkera M.P., Burckhardb J., Mittsc D.A., Williamsd K.B.: Dimensional change over time of extended-storage alginate impression materials, Angle Orthod., 2010, 80:1110-1115.

20- Sedda M., Casarotto A., Rausita A., Borracchini A.: Effect of storage time on the accuracy of casts made from different irreversible hydrocolloids, The Journal of Contemporary Dental Practice, 2008, 9(4):178-186. 